
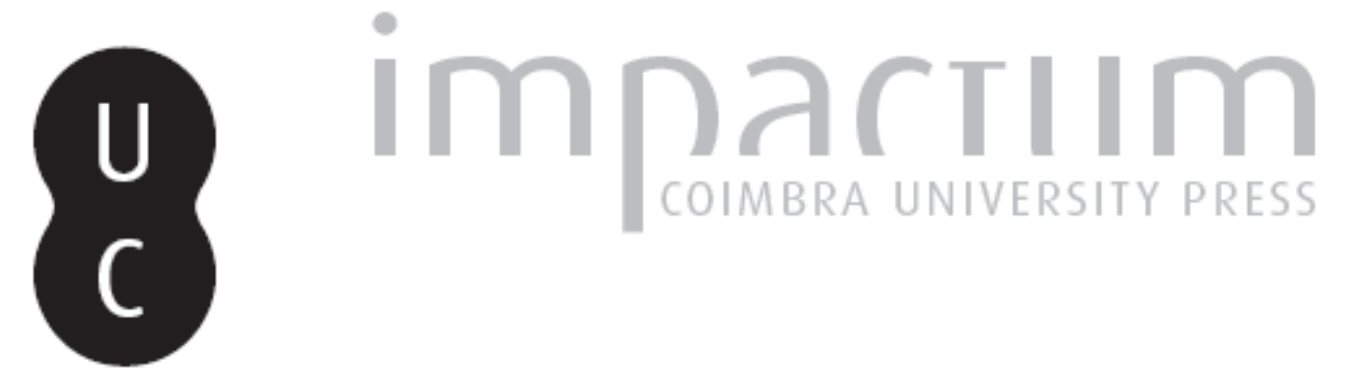

Abaciológio do mosteiro de S. Vicente de Vacariça (séc. XI)

Autor(es): $\quad$ Gouveia, Mário de

Publicado por: Centro de História da Sociedade e da Cultura

URL persistente:

URI:http://hdl.handle.net/10316.2/39424

DOI:

DOI:http://dx.doi.org/10.14195/1645-2259_12_3

Accessed : $\quad$ 26-Apr-2023 08:12:12

A navegação consulta e descarregamento dos títulos inseridos nas Bibliotecas Digitais UC Digitalis, UC Pombalina e UC Impactum, pressupõem a aceitação plena e sem reservas dos Termos e Condições de Uso destas Bibliotecas Digitais, disponíveis em https://digitalis.uc.pt/pt-pt/termos.

Conforme exposto nos referidos Termos e Condições de Uso, o descarregamento de títulos de acesso restrito requer uma licença válida de autorização devendo o utilizador aceder ao(s) documento(s) a partir de um endereço de IP da instituição detentora da supramencionada licença.

Ao utilizador é apenas permitido o descarregamento para uso pessoal, pelo que o emprego do(s) título(s) descarregado(s) para outro fim, designadamente comercial, carece de autorização do respetivo autor ou editor da obra.

Na medida em que todas as obras da UC Digitalis se encontram protegidas pelo Código do Direito de Autor e Direitos Conexos e demais legislação aplicável, toda a cópia, parcial ou total, deste documento, nos casos em que é legalmente admitida, deverá conter ou fazer-se acompanhar por este aviso.

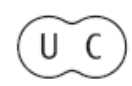


evista de História da Sociedade e da Cultura

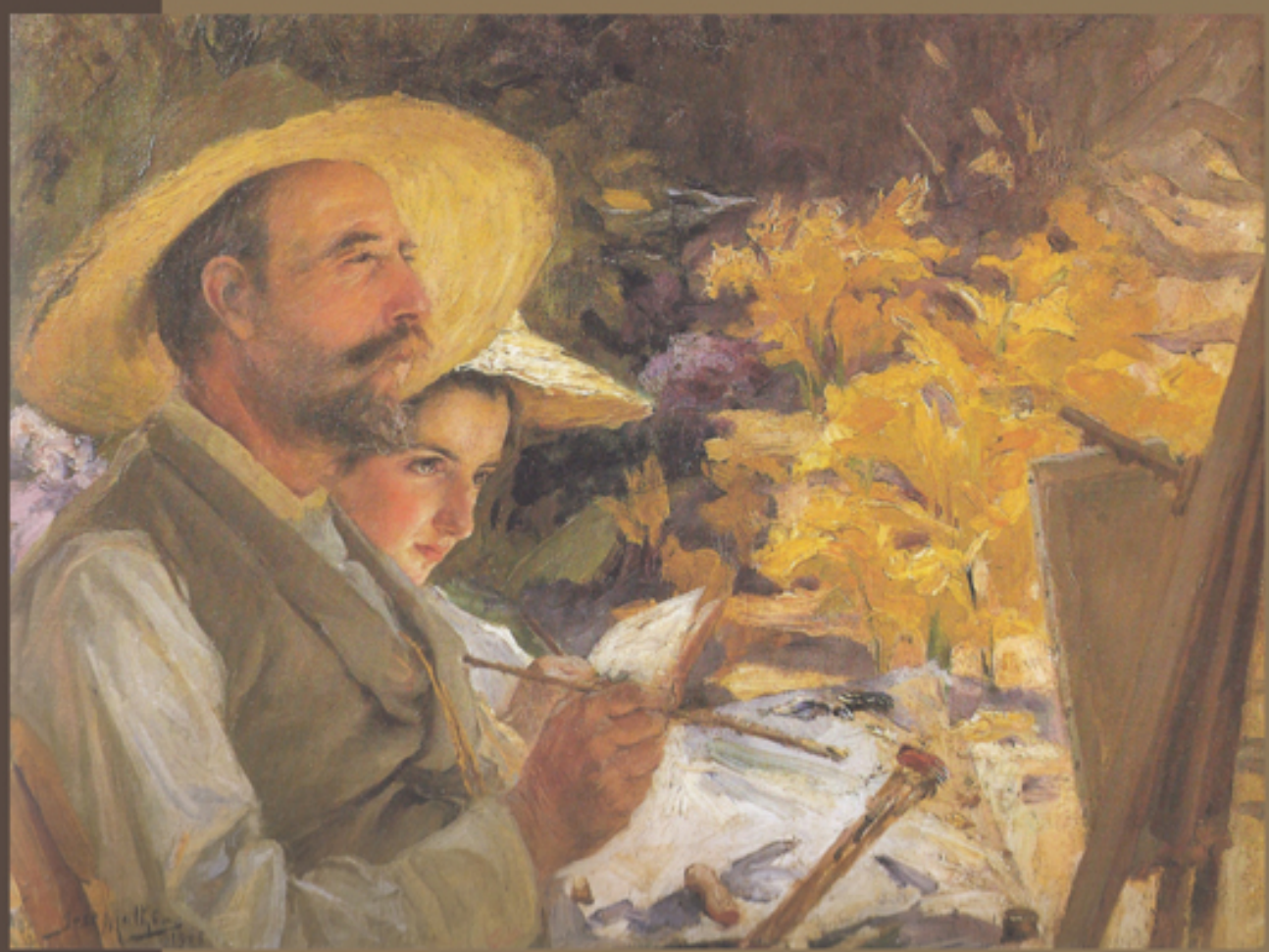

Centro de História da Sociedade e da Cultura Universidade de Coimbra 


\title{
Abaciológio do mosteiro de S. Vicente de Vacariça (séc. XI)
}

\author{
Mário de Gouveia \\ Instituto de Estudos Medievais da FCSH da UNL \\ gouveia.mnc@gmail.com \\ Texto recebido em /Text submitted on: 06/12/2011 \\ Texto aprovado em /Text approved on: 08/02/2012
}

Resumo/Abstract:

O objectivo do presente ensaio é apresentar uma reconstituição da sucessão abacial do mosteiro de S. Vicente de Vacariça durante o século XI, a partir da análise dos títulos diplomáticos reunidos no cartulário medieval da Sé de Coimbra conhecido pela designação de Livro preto.

The aim of this essay is to present a recomposition of the abbatial succession of the monastery of Saint Vincent of Vacariça during the $11^{\text {th }}$ century, based upon the analysis of the diplomatic acts compilated in the medieval cartulary of Coimbra's Cathedral known as Livro preto.

Palavras chave/Keywords:

Alta Idade Média (séc. XI); Diplomática; Livro preto da Sé de Coimbra; Mosteiro de S. Vicente de Vacariça; Abades.

High Middle Ages (11 ${ }^{\text {th }}$ century); Diplomatics; Livro preto of Coimbra's Cathedral; Monastery of Saint Vincent of Vacariça; Abbots. 


\section{Advertência prévia}

As informações de que dispomos para reconstituir o abaciológio do mosteiro de S. Vicente de Vacariça baseiam-se principalmente na crítica interna dos diplomas reunidos, sob a forma de cópias, no Livro preto da Sé de Coimbra ${ }^{1}$. O cartulário diocesano, cuja elaboração remonta aos séculos XII e XIII, contém várias centenas de diplomas relativos maioritariamente à história da sede catedralícia, bem como das instituições monásticas e eclesiásticas que integraram o respectivo sistema de padroado². Nessa qualidade, a produção do cartulário deve ser vista como uma expressão da necessidade de se salvaguardarem e transmitirem para a posteridade os títulos jurídicos probatórios dos direitos patrimoniais e dominiais adquiridos por estas instâncias ao longo da sua história, numa cronologia que abrange sensivelmente os séculos IX a XIII.

$\mathrm{Na}$ sua generalidade, os diplomas que compõem o fundo arquivístico do mosteiro de Vacariça chegaram até nós maioritariamente sob a forma de apógrafos - e alguns raríssimos autógrafos ${ }^{3}$ - transcritos e reunidos,

1 Exclui-se neste passo a crítica externa, na medida em que as informações a que nos referimos provêm maioritariamente de documentos conservados, sob a forma de cópias, no Livro preto da Sé de Coimbra. Este facto impede-nos de tecer considerações sobre algumas problemáticas de natureza técnica, relacionadas com as características diplomáticas e paleográficas dos respectivos originais, que não chegaram até nós.

2 Sobre a actividade de produção diplomática na chancelaria da sede catedralícia, leia-se, por todos, MORUJÃO, Maria do Rosário Barbosa - A Sé de Coimbra. A instituição e a chancelaria (1080-1318). Lisboa: Fundação Calouste Gulbenkian - Fundação para a Ciência e a Tecnologia/Ministério da Ciência, Tecnologia e Ensino Superior, 2010, em especial p. 293-679 (sintetizado em CUNHA, Maria Cristina Almeida e; SARAIVA, Anísio Miguel de Sousa e MORUJÃO, Maria do Rosário Barbosa - Traditionalisme, régionalisme et innovation dans les chancelleries épiscopales portugaises au Moyen Âge, in Régionalisme et internationalisme. Problèmes de paléographie et de codicologie au Moyen Âge [Actes du $X V^{e}$ Colloque du Comité International de Paléographie Latine <Vienne, 13-17 Septembre 2005>; édités par Otto Kresten et Franz Lackner], s.l., Verlag der Österreichischen Akademie der Wissenschaften, 2008, p. 299-316, em especial p. 309-312). Especificamente sobre o Livro preto da Sé de Coimbra, leia-se MORUJÃO, Maria do Rosário Barbosa - O Livro preto da Sé de Coimbra. Estudo do cartulário, Revista de história da sociedade e da cultura, n. ${ }^{\circ} 8,2008$, p. $7-43$.

3 Identificados e descritos em COSTA, Avelino de Jesus da - Coimbra - centro de atracção e de irradiação de códices e de documentos, dentro da Península, nos sécs. XI e XII, in Actas das II Jornadas luso-espanholas de história medieval, vol. IV, Porto, Instituto Nacional de Investigação Científica, 1990, p. 1309-1334, em especial p. 1313. Segundo 
sem solução de continuidade, neste cartulário diocesano. Correspondem geralmente a actos da prática que podemos identificar como cartas de doação, de compra e venda ou de permuta, ou ainda como inventários de bens e propriedades e outros mais raros documentos de tipologia diversa, como uma epístola. Embora a sua cópia para o cartulário onde hoje se conservam tenha ocorrido posteriormente, acabaram por integrar o fundo arquivístico da Sé de Coimbra depois da redução do mosteiro de Vacariça à condição de igreja do padroado diocesano, por doação dos condes Raimundo e Urraca, em $1094^{4}$.

As datas que podemos propor para a reconstituição dos sucessivos períodos de governo dos abades vacaricenses resultam, portanto, da análise de diplomas que foram produzidos com uma finalidade essencialmente administrativa, e, nestas circunstâncias, não têm quaisquer relações com outras tipologias de fontes relacionadas com aquela questão, como os nomina abaciais que encontramos num registo transcrito no verso do fólio de guarda do Livro dos testamentos do mosteiro de S. Mamede de Lorvão, datável da segunda década do século XII ${ }^{5}$.

este investigador, conservam-se actualmente nos AN/TT os seguintes autógrafos: AN/TT, Sé de Coimbra, mç. I, docs. 7, 10, 11 e 38 (as referências não foram por nós cotejadas com os pergaminhos aí depositados).

4 LP 82 (1094.11.13).

5 AN/TT, Ordem de Cister, Mosteiro de Lorvão, liv. 49 - ref. a PT-TT-MSML/49. Trata-se de uma relação em que se procede à enumeração dos nomes dos dez abades ([Nomina abbatum]) que administraram o mosteiro pelo menos entre os inícios do século X e os inícios do XII, de Justo a Eusébio. Para além de conter uma referência ao momento em que se deu a restauração da autonomia institucional do mosteiro, na sequência da sua desvinculação da instituição padroeira, a igreja de St. ${ }^{a}$ Maria da Sé de Coimbra, em 1116, e da ordenação de Eusébio como seu novo abade, num contexto de oposição e resistência do colectivo religioso à política reformadora desenvolvida pelas autoridades leonesas e francesas em Coimbra, o registo remete-nos fundamentalmente para as datas de ocorrência do óbito de cada abade. No suporte material original, esta relação antecede um outro registo formado por seis ementas analísticas relativas ao período que decorre de 866 a 1110 ([1109]), bem como outra primeira relação alusiva aos nomes de três reis leoneses ([Nomina regum]) - Ramiro II, Sancho I e Bermudo II - que desempenharam um significativo papel na consolidação do património monástico durante o século X. O texto contendo os nomes dos dez abades foi alvo de várias publicações, pelo menos desde a primeira metade do século XVIII: ROCHA, Fr. Manoel da - Portugal renascido. Tratado historico-critico-chronologico em que à luz da verdade se dão manifestos os successos de Portugal do seculo decimo depois do nascimento de Christo Senhor Nosso. Lisboa ocidental: Off. de Joseph Antonio da Sylva, 1730, p. 147; RIBEIRO, João Pedro - Dissertações chronologicas e criticas sobre a historia e a jurisprudencia 
O universo documental disponível permite-nos situar e contextualizar a acção dos diversos abades vacaricenses na escala do tempo monástico com alguma margem de segurança. Conhecemos a linha de sucessão dos abades que administraram o mosteiro ao longo do século XI, mas não somos capazes de fixar datas exactas para o início e o termo do exercício das funções abaciais. Esta limitação deve-se sobretudo à natureza das fontes que chegaram até nós, que, conforme salientámos anteriormente, não foram produzidas com o objectivo de nos dar informações sobre esta problemática específica do foro institucional. As fontes reflectem acima de tudo as principais linhas da política de aquisição, gestão e alienação de bens e propriedades pela comunidade monástica ao longo daquele século, ou seja, reportam-se à dinâmica económica que o colectivo religioso procurou desenvolver visando a sua própria sobrevivência institucional. Não obstante o facto de os diversos abades conhecidos surgirem à frente da comunidade monástica como agentes impulsionadores desta dinâmica ou no exercício de outras prerrogativas próprias da sua condição abacial, nada se nos é dado a conhecer acerca das circunstâncias que enquadraram a sua ordenação ou das razões que levaram ao seu afastamento do exercício das funções abaciais.

Em termos cronológicos, os dados extraídos das fontes têm um carácter aproximado, e, nessa qualidade, devem entender-se apenas como pontos de referência para o enquadramento temporal dos sucessivos períodos de governo abacial no seio da comunidade monástica. Com base nos diplomas do mosteiro de Vacariça e de outros mosteiros do Entre-Minho-e-Mondego

ecclesiastica e civil de Portugal, t. IV, parte I. Lisboa: Na Typografia da Mesma Academia [R. das Sciencias de Lisboa], 1819, p. 175, apênd. II; SS, vol. I, p. 20; CÁCEGAS, Luís de - Idade Média. Notas de história e de crítica, Brotéria, vol. XXI, fasc. IV, Julho de 1923 , p. 277-278; Liber testamentorum coenobii laurbanensis (Estudios). Leão: Centro de Estudios e Investigación «San Isidoro» - Caja España de Inversiones - Archivo Histórico Diocesano, 2008, p. 591-592 (cf. reprodução comentada do texto nas p. 465-466). Mais recentemente, acompanhado de um alargado estudo sobre a analística monástica portucalense dos séculos XI e XII, também em GOUVEIA, Mário Nuno Campos de - O limiar da tradição no moçarabismo conimbricense. Os Anais de Lorvão e a memória monástica do território de fronteira (séc. IX-XII). Lisboa: 2008, p. 371-378 (anexo 1, reproduzindo SS, vol. I, p. 20) (Edição policopiada da Dissertação apresentada para cumprimento dos requisitos necessários à obtenção do grau de Mestre em História, na Área de Especialização em História Medieval, pela Faculdade de Ciências Sociais e Humanas da Universidade Nova de Lisboa, realizada sob a orientação científica dos Professores Doutor José João da Conceição Gonçalves Mattoso e Doutor Luís Filipe Llach Krus). 
que integraram nos séculos XI e XII o seu padroado, podemos afirmar terem existido pelo menos sete figuras seguramente identificáveis como abades da comunidade monástica ${ }^{6}$. Aqueles que desempenharam esta função e que se encontram historicamente documentados são, por ordem cronológica de outorga do diploma que atesta pela primeira vez essa condição: André $(1002)^{7}$, Emilião $(1016)^{8}$, Tudeíldo $(1018)^{9}$, Flórido (1036) ${ }^{10}$, Alvito $(1047)^{11}$, Ramiro (1086) ${ }^{12}$ e Salomão (1090) ${ }^{13}$. Todos estes abades surgem na documentação conservada no Livro preto da Sé de Coimbra como superiores hierárquicos da comunidade monástica. $\mathrm{O}$ único caso que suscita reservas

6 A «tabela ideográfico-sistemática» da mais recente edição do Livro preto da Sé de Coimbra (pub. em Livro preto. Cartulário da Sé de Coimbra [Edição crítica; texto integral; director e coordenador editorial: Manuel Augusto Rodrigues; director científico: Cónego Avelino de Jesus da Costa], Coimbra, Arquivo da Universidade de Coimbra, 1999, p. 1174) contém algumas incorrecções no respeitante à fixação, em índice remissivo, dos nomes dos abades vacaricenses. Esta edição do cartulário diocesano identifica mais dois abades - Salvado e Tiago -, para além de Alvito, André, Emilião, Flórido, João, Ramiro, Salomão e Tudeíldo, os únicos que estão seguramente documentados nos diplomas monásticos. Não é possível relacionar aqueles dois abades com o mosteiro de Vacariça com base na documentação subsistente. Salvado não aparece em nenhum documento conservado no cartulário como superior hierárquico do mosteiro. $\mathrm{O}$ mesmo pode dizer-se a propósito de Tiago. Neste caso, a impossibilidade de se relacionar o abade com o mosteiro de Vacariça é ainda mais flagrante: em primeiro lugar, devemos ter em conta as datas dos documentos em que Tiago é mencionado, demasiado recuadas relativamente às primeiras notícias que se conservam acerca da igreja (972) ou do mosteiro de Vacariça (1002); em segundo lugar, devemos sublinhar o facto de Tiago figurar com o título de abade em documentos relativos ao mosteiro de St. ${ }^{\circ}$ André de Sever do Vouga, o qual, aliás, veio a integrar o padroado do mosteiro de Vacariça. Estes documentos afirmam que Tiago terá começado a desempenhar funções abaciais depois de os proprietários dos direitos sobre aquele mosteiro lho terem legado, vindo este a regressar novamente às mãos de particulares dada a inexistência de descendentes. Nas entradas do índice onomástico da mais recente edição do cartulário, apenas é citado Tiago - alegadamente documentado como abade vacaricense em LP 134 e LP 139 -, sem se fazer qualquer referência a Salvado. Deste índice constam também outras incorrecções quanto à documentação que supostamente refere os restantes abades vacaricenses: ao contrário do que se afirma, não há qualquer referência a Tudé́ldo em LP 91 e LP 156. No primeiro destes diplomas, para além de não figurar nenhuma personagem com este nome, quem é aí citado como abade é André.

7 LP $126(1002.11 .30)$.

8 LP 124 (1016.02.10).

9 LP 129 e 161 (1018.01.30).

${ }^{10}$ LP 93 e 146 (1036.02.22).

${ }^{11}$ LP 130 e 132 (1047.10.12 ou 13).

${ }^{12}$ LP 372 (1086.08.11).

${ }^{13}$ LP 255 (1090.08.16). 
é o de João (1047) ${ }^{14}$. Alguns identificam-se também como abades de outras comunidades residentes nos mosteiros do padroado vacaricense, como Tudeíldo, relativamente aos mosteiros de S. Salvador de Leça ${ }^{15}$, S. Martinho de Anta $^{16}$ e S. Martinho de Aldoar ${ }^{17}$; e Salomão, relativamente ao de Trezói ${ }^{18}$.

As datas apontadas para cada abade correspondem apenas à data da primeira referência documental em que estas figuras surgem no desempenho das funções abaciais. Não correspondem, portanto, ao ano da ordenação de cada abade. O mesmo pode dizer-se a propósito das datas em que cada abade é citado pela última vez. Estas não reflectem necessariamente o momento em que se deu a sua morte ou o seu afastamento do cargo. Tenha-se em conta o caso de Tudé́ldo: embora citado como abade do mosteiro de Vacariça desde $1018^{19}$, deslocou-se para o mosteiro de Leça em 1026 - a primeira referência documental nesta qualidade remonta apenas a $1032^{20}$ - e passou a governar esta comunidade monástica até à sua morte em 1046, deixando atrás de si um prioste, Flórido, no uso do título de abade desde $1036^{21}$. Durante a sua estadia em Leça, Tudeíldo é citado igualmente como abade de Anta, desde $1037^{22}$, e de Aldoar, desde $1044^{23}$. Poucos meses antes de falecer, Tudeíldo preparou a sua sucessão nos mosteiros dependentes de Vacariça. Em 1045 24 ainda foi designado como abade de Vacariça, e o seu representante neste mosteiro, novamente como prioste. Este desdobramento da condição abacial voltará a verificar-se mais tarde com outras figuras da hierarquia monástica: Flórido desempenhou funções como prioste de Tudeíldo; Alvito, de Flórido; Ildras, de Alvito; Cid, de Ramiro; e Godinho, de Salomão.

\footnotetext{
${ }^{14}$ LP $86(1047.12 .20)$.

${ }^{15}$ LP 353 (1032.03.13), LP 157 (1032.04.24), LP 189 (1032.07.31), LP 190 (1034.07.21), LP 184 (1035.02.23), LP 516 (1035.03.28), LP 362 e 511 (1037.03.14), LP 198 (1039.04.22), LP 512 (1041.02.28), LP 192 (1045.06.06), LP 520 (1046.01.29) e LP 369 (1046.08.14).

${ }^{16}$ LP 367 (1037.08.04), LP 186 (1038.04.01), LP 363 (1041.04.19) e LP 521 (1043.05.20).

${ }^{17}$ LP 152 (1044.07.22).

${ }^{18}$ LP 77 (1098.12.17).

${ }^{19}$ LP 129 e $161(1018.01 .30)$.

${ }^{20}$ LP 353 (1032.03.13).

${ }^{21}$ LP 93 e 146 (1036.02.22).

${ }^{22}$ LP 367 (1037.08.04).

${ }^{23}$ LP 152 (1044.07.22).

${ }^{24}$ LP 138 e 150 (1045.09.21).
} 
Ao longo do século XI, o título mais utilizado pelos superiores do mosteiro de Vacariça foi o de abbas, que, à excepção de João, se encontra associado a todos os abades. O primeiro a utilizá-lo foi André, e o último, Salomão. De André a Salomão, a sequência de utilização do título é ininterrupta, embora se registando uma clara tendência para a acumulação sucessiva de títulos: André, Emilião e Tudeíldo apenas usam um título (abbas); mas Flórido recorre já a dois (abbas e praepositus); Alvito e Ramiro, a três (abbas, praepositus e presbyter, no primeiro caso; abbas, ducator e prior, no segundo); e Salomão, a cinco (abbas, rector, praepositus, prior e presbyter). Com Alvito, Ramiro e Salomão - ou seja, os três abades que antecederam a extinção do mosteiro de Vacariça -, o abade é também identificado como clérigo, mais concretamente como um presbítero ou, mais tarde, como um prior. O número de abades que desempenharam funções como clérigos foi, pois, menos significativo do que o relacionado exclusivamente com o desempenho de funções abaciais: verifica-se na documentação vacaricense uma clara primazia do exercício da função abacial sobre a função clerical. Este facto, conjugado com a cronologia tardia das referências documentais, sugere uma maior proximidade de Ramiro e Salomão, os dois últimos abades do mosteiro de Vacariça, às directrizes da reforma religiosa ocorrida na Hispânia cristã nos finais do século XI, que passou a preconizar o alargamento das funções clericais no seio das congregações religiosas tradicionais à figura abacial.

A título de conclusão, registe-se, por fim, que cada entrada é composta por um conjunto de sete descritores, sob a forma de alíneas alfabeticamente ordenadas de (a) a (g): na primeira (a), procede-se à identificação das referências documentais a cada abade, por título; na segunda (b), à discriminação da geografia do património adquirido por cada abade, por modalidade jurídica de aquisição; na terceira (c), à identificação dos direitos de padroado adquiridos por cada abade; na quarta (d), à discriminação das expressões relativas a actos de produção documental per manum propriam; na quinta (e), à identificação das expressões relativas a actos de subscrição documental per manum propriam; na sexta (f), à identificação dos clérigos-notários associados a cada abade; e, por fim, na sétima (g), à identificação de todas as referências documentais a cada abade, por ordem cronológica de outorga dos diplomas que se lhe referem nessa qualidade. 


\section{Abaciológio}

1. ANDRÉ

a) Referências documentais, por título: abbas: LP 126 (1002.11.30), LP 135 (1005.12.13) e LP 91 (1020.12.01); título não-referido: LP 72 (1006.06.17).

b) Geografia do património adquirido durante o seu abaciado: por doação: Penso (conc. de S. Pedro do Sul, dist. de Viseu) (LP 126, de 1002.11.30); Vila Nova de Monsarros (conc. de Anadia, dist. de Aveiro) (LP 72, de 1006.06.17); Levira (conc. de Oliveira do Bairro, dist. de Aveiro) e Lázaro (conc. de Águeda, dist. de Aveiro) (LP 91, de 1020.12.01); por compra: doc. inexistente; por permuta: doc. inexistente.

c) Direitos de padroado adquiridos durante o seu abaciado: mosteiro de S. Paio de Rocas do Vouga (conc. de Sever do Vouga, dist. de Aveiro) (LP 126, de 1002.11.30).

d) Expressões relativas a actos de produção documental per manum propriam: scripsit: doc. inexistente.

e) Expressões relativas a actos de subscrição documental per manum propriam: adfuit: doc. inexistente; conf: LP 135 (1005.12.13); test.: doc. inexistente.

f) Clérigos-notários documentados durante o seu abaciado:

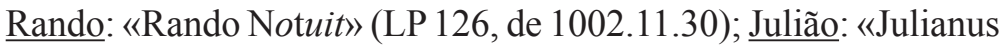
presbiter Notuit» (LP 72, de 1006.06.17); Zalama: «Zalama presbiter Notuit» (LP 91, de 1020.12.01).

g) Referências documentais, por ordem cronológica: LP 126 (1002.11.30), LP 135 (1005.12.13), LP 72 (1006.06.17) e LP 91 (1020.12.01). 


\section{EMILIÃo}

a) Referências documentais, por título: abbas: LP 124 (1016.02.10), LP 123 (1018.01.12) e LP 122 (1018).

b) Geografia do património adquirido durante o seu abaciado: por doação: Recardães (conc. de Águeda, dist. de Aveiro) (LP 124, de 1016.02.10); por compra: Recardães (conc. de Águeda, dist. de Aveiro) (LP 122, de 1018); por permuta: doc. inexistente.

c) Direitos de padroado adquiridos durante o seu abaciado: igreja de S. Miguel de Recardães (conc. de Águeda, dist. de Aveiro) (LP 123, de 1018.01.12).

d) Expressões relativas a actos de produção documental per manum propriam: scripsit: doc. inexistente.

e) Expressões relativas a actos de subscrição documental per manum propriam: adfuit: doc. inexistente; conf: doc. inexistente; test.: doc. inexistente.

f) Clérigos-notários documentados durante o seu abaciado: Rodrigo: «Rodericus exarauit memorie» (LP 123, de 1018.01.12); clérigos-notários não-referidos: LP 124 (1016.02.10) e LP 122 (1018).

g) Referências documentais, por ordem cronológica: LP 124 (1016.02.10), LP 123 (1018.01.12) e LP 122 (1018).

3. TudeíldDo

a) Referências documentais, por título: abbas: LP 129 e 161 (1018.01.30), LP 120 (1018), LP 121 (1019.02.28), LP 134 (1019.12.02), LP 142 (1021.11.20), LP 144 (1023.09.21), LP 143 ([1023.09.21]), LP 359 (1025.09.21), LP 109 ([1026-1045]), LP 
353 (1032.03.13), LP 157 (1032.04.24), LP 189 (1032.07.31), LP 145 (1034.02.10), LP 190 (1034.07.21), LP 184 (1035.02.23), LP 516 (1035.03.28), LP 362 e 511 (1037.03.14), LP 367 (1037.08.04), LP 185 (1038.04.01), LP 186 (1038.04.01), LP 198 (1039.04.22), LP 115 e 140 (1040.08.13), LP 512 (1041.02.28), LP 363 (1041.04.19), LP 521 (1043.05.20), LP 152 (1044.07.22), LP 192 (1045.06.06), LP 137 e 148 (1045.09.21), LP 138 e 150 (1045.09.21), LP 153 (1045.09.21), LP 520 (1046.01.29) e LP 369 (1046.08.14); título não-referido: LP 512 (1041.02.28).

Obs.: Tudeíldo é tio de Randulfo, clérigo-notário do mosteiro de Vacariça, abade do mosteiro de Leça e possivelmente secretário do bispo Sesnando do Porto durante o Concílio de Coiança, celebrado em 1055. Deve-se a este clérigo-notário uma das cópias dos decretos do referido Concílio, conservada no Livro preto da Sé de Coimbra (LP 567, de [1055]: «Hoc decretum factum fuit in concilio domni Fernandi regis te [sic] sue conjugis regine domne Sancie in urbe Cogianca. Et adduxit inde illud Randulfus presbiter de acisterio Vaccariza pro memoria posteris.»), considerada por vários investigadores como a mais fidedigna que subsistiu até à actualidade. Durante o abaciado de Tudeíldo, Flórido desempenhou funções como prioste no mosteiro de Vacariça (LP 138 e 150, de 1045.09.21). Pouco antes de falecer, Tudeíldo preparou a sua sucessão nas funções abaciais a favor de Randulfo, no mosteiro de Leça, e de Pedro, possivelmente no de Anta.

Como abade do mosteiro de Vacariça: LP 129 e 161 (1018.01.30), LP 120 (1018), LP 121 (1019.02.28), LP 134 (1019.12.02), LP 142 (1021.11.20), LP 144 (1023.09.21), LP 143 ([1023.09.21]), LP 359 (1025.09.21), LP 145 (1034.02.10), LP 115 e 140 (1040.08.13), LP 137 e 148 (1045.09.21), LP 138 e 150 (1045.09.21) e LP 153 (1045.09.21).

Como abade do mosteiro de Leça: LP 353 (1032.03.13), LP 157 (1032.04.24), LP 189 (1032.07.31), LP 190 (1034.07.21), LP 184 
(1035.02.23), LP 516 (1035.03.28), LP 362 e 511 (1037.03.14), LP 198 (1039.04.22), LP 512 (1041.02.28), LP 192 (1045.06.06), LP 520 (1046.01.29) e LP 369 (1046.08.14).

Como abade do mosteiro de Anta: LP 367 (1037.08.04), LP 185 (1038.04.01), LP 186 (1038.04.01), LP 363 (1041.04.19) e LP 521 (1043.05.20).

Como abade do mosteiro de Aldoar: LP 152 (1044.07.22).

b) Geografia do património adquirido durante o seu abaciado: por doação: Paradela (conc. de Sever do Vouga, dist. de Aveiro), Aboim (conc. de Sever do Vouga, dist. de Aveiro), Nespereira (conc. de Oliveira de Azeméis, dist. de Aveiro), Sá ou S. Martinho (conc. de Sever do Vouga, dist. de Aveiro) e Espinhel (conc. de Águeda, dist. de Aveiro) (LP 129 e 161, de 1018.01.30; Nespereira e Espinhel mencionadas apenas em LP 129; Paradela e Aboim mencionadas apenas em LP 161); Sever do Vouga (conc. de Sever do Vouga, dist. de Aveiro), Quintela (conc. de Sever do Vouga, dist. de Aveiro), Castelões (conc. de Sever do Vouga, dist. de Aveiro), Cambra (conc. de Vale de Cambra, dist. de Aveiro) e Pessegueiro do Vouga (conc. de Sever do Vouga, dist. de Aveiro) (LP 121, de 1019.02.28); S. Gens (conc. de Matosinhos, dist. do Porto), Patrocello (loc. incerta), Saltarios (loc. incerta), Recarei (conc. de Matosinhos, dist. do Porto), Kaeiros (loc. incerta), Moalde (conc. de Matosinhos, dist. do Porto), Pousadela (loc. incerta), Sevilhães (conc. de Gondomar, dist. do Porto), Medancelhe (conc. de Gondomar, dist. do Porto), Gondarém (conc. do Porto, dist. do Porto), Vilar (conc. de Gondomar, dist. do Porto), Coritelo (conc. da Maia, dist. do Porto), Villar Tora Durio (loc. incerta), Aldoar (conc. do Porto, dist. do Porto) (LP 142, de 1021.11.20; cf., in genere, as doações constantes de LP 147; cf., para Sevilhães, LP 523); Gatões (conc. de Matosinhos, dist. do Porto) (LP 189, de 1032.07.31); Leoueriz (loc. incerta) (LP 190, de 1034.07.21); Paranhos (conc. do Porto, dist. do Porto) e Cauno (loc. incerta) 
(LP 184, de 1035.02.23); Leça (conc. de Matosinhos, dist. do Porto), S. Gens (conc. de Matosinhos, dist. do Porto), Patrocello (loc. incerta), Salgarios (loc. incerta), Recarei (conc. de Matosinhos, dist. do Porto), Queiranos (loc. incerta), Villa Flamulini (loc. incerta), Villa Mundini (loc. incerta), Vermoim (conc. da Maia, dist. do Porto), Aldoar (conc. do Porto, dist. do Porto), Lordelo (conc. de Vale de Cambra, dist. de Aveiro), Vila Chã (conc. de Vale de Cambra, dist. de Aveiro), Pinheiro (conc. de Castelo de Paiva, dist. de Aveiro), Sevilhães (conc. de Gondomar, dist. do Porto), Medancelhe (conc. de Gondomar, dist. do Porto), Moalde (conc. de Matosinhos, dist. do Porto), Pousadela (loc. incerta), Pedrouços (conc. da Maia, dist. do Porto), Coroado (conc. de St. ${ }^{o}$ Tirso, dist. do Porto), Villa de Lillia (loc. incerta), Refojos (conc. de St. ${ }^{\circ}$ Tirso, dist. do Porto), Villa de Osonio (loc. incerta), Ville Ofreiso (loc. incerta), Marentães (conc. de Vila Nova de Gaia, dist. do Porto) (LP 147, de [1035].12.04; com a respectiva confirmação em LP 115 e 140; cf., in genere, as doações constantes de LP 142); Pousada (conc. de St. ${ }^{a}$ Maria da Feira, dist. de Aveiro) e St. ${ }^{a}$ Cruz (conc. de Espinho, dist. de Aveiro) (LP 367, de 1037.08.04); Anta (conc. de Espinho, dist. de Aveiro) (LP 185, de 1038.04.01); Real (conc. de Matosinhos, dist. do Porto) e Gondivai (conc. de Matosinhos, dist. do Porto) (LP 198, de 1039.04.22); localidades não-especificadas (LP 512, de 1041.02.28); Pousada (conc. de St. ${ }^{a}$ Maria da Feira, dist. de Aveiro) e St. ${ }^{a}$ Cruz (conc. de Espinho, dist. de Aveiro) (LP 521, de 1043.05.20); Fontela (loc. incerta) e Vale (loc. incerta) (LP 152, de 1044.07.22); Leça (conc. de Matosinhos, dist. do Porto) (LP 192, de 1045.06.06; cf. LP 137 e 148, LP 138 e 150); Coroado (conc. de St. ${ }^{\circ}$ Tirso, dist. do Porto) e Rebordões (conc. de St. ${ }^{\circ}$ Tirso, dist. do Porto) (LP 369, de 1046.08.14); por compra: Recardães (conc. de Águeda, dist. de Aveiro) (LP 120, de 1018); Sever do Vouga (conc. de Sever do Vouga, dist. de Aveiro) e Quintela (conc. de Sever do Vouga, dist. de Aveiro) (LP 144, de 1023.09.21; cf. LP 143; com registo de demarcação das propriedades adquiridas em LP 109); Bouças (conc. de Matosinhos, dist. do Porto) (LP 157, de 1032.04.24); Leça (conc. de Matosinhos, dist. do Porto) 
e Pinheiro (conc. da Maia, dist. do Porto) (LP 520, de 1046.01.29); por permuta: doc. inexistente.

Obs.: Tudeíldo esteve na posse de bens em Pousada e Custóias, tal como de algumas salinas na foz do Leça, ainda antes de 1045 (LP 137 e 148, de 1045.09.21; LP 138 e 150, de 1045.09.21). Nãoé possível saber ao certo a data em que algumas destas propriedades foram integradas no património do mosteiro de Vacariça. Desconhecemos também quando foi adquirida pelo mosteiro uma casa em Rocas do Vouga, cujos direitos de posse foram litigados pelo abade e os presbíteros Guterre e Bermudo, em 1025 (LP 359, de 1025.09.21). É possível que se identifique com este último o presbítero homónimo que se compromeu, junto com o presbítero Froila, a coabitar o mosteiro de Rocas do Vouga sob a supervisão de Tudé́ldo, em 1034 (LP 145, de 1034.02.10).

\section{c) Direitos de padroado adquiridos durante o seu abaciado:} mosteiro de St. ${ }^{\circ}$ André de Sever do Vouga (conc. de Sever do Vouga, dist. de Aveiro) (integração definitiva em LP 134, de 1019.12.02; cf. LP 129 e 161); mosteiro de S. Salvador de Leça (conc. de Matosinhos, dist. do Porto), igreja de S. Martinho de Aldoar (conc. do Porto, dist. do Porto) e igreja de S. Mamede de Moalde (conc. de Matosinhos, dist. do Porto) (LP 142, de 1021.11.20; os direitos sobre a igreja de Aldoar foram contestados em 1041 pela condessa Ilduara Mendes, vindo esta a reconhecer ao abade a legitimidade das suas reivindicações em sede de litígio; cf., a propósito, LP 363; cf., sobre o padroado de Leça e Aldoar, LP 137 e 148); mosteiro de S. Romão de Vermoim (conc. da Maia, dist. do Porto), igreja de S. Mamede de Coronado (conc. da Trofa, dist. do Porto), igreja de S. Tiago de [Jovim] (conc. de Gondomar, dist. do Porto) e igreja de S. Paio de Marentães (conc. de Vila Nova de Gaia, dist. do Porto) (LP 147, de [1035].12.04).

Obs.: É possível que date do abaciado de Tudeíldo o procedimento de incorporação de direitos sobre alguns dos mosteiros e igrejas 
referidos na «Noticia de uillis uacaricie» (LP 73, de 1064), pese embora o facto de, como registo de inventário do património monástico situado no Entre-Vouga-e-Mondego à data da conquista de Coimbra por Fernando I, em 1064, este instrumento ter sido elaborado por um dos seus sucessores, provavelmente Alvito. Tudeíldo foi proprietário, a título pessoal, dos mosteiros de Leça, Anta e Vermoim, que doou ao de Vacariça durante o governo do seu prioste, Flórido, poucos meses antes de falecer (LP 138 e 150, de 1045.09.21).

d) Expressões relativas a actos de produção documental per manum propriam: scripsit: doc. inexistente.

e) Expressões relativas a actos de subscrição documental per manum propriam: adfuit: doc. inexistente; conf: LP 516 (1035.03.28), LP 362 e 511 (1037.03.14) e LP 137 e 148 (1045.09.21); test.: doc. inexistente.

f) Clérigos-notários documentados durante o seu abaciado: Mendo: «Menendus presbiter Notuit» (LP 142, de 1021.11.20); Frutuoso: «Fructuosi notuit» (LP 359, de 1025.09.21); $\underline{\text { Ansemundo: }}$ «Ansemondo scipsit» (LP 109, de [1026-1045]; «Ansemondus notuit» (LP 184, de 1035.02.23); «Ansemondo quasi presbiter notuit» (LP 367, de 1037.08.04); «Johannes presbiter denuntiavit, $\mathrm{A}[n]$ semundus presbiter quos exaravit» (LP 185, de 1038.04.01); «Ansemundus notuit» (LP 186, de 1038.04.01); «Ansemundus notuit» (LP 198, de 1039.04.22); «Ansemondu notuit» (LP 512, de 1041.02.28); «Ego ansemondus Quos exarauj» (LP 138 e 150, de 1045.09.21); João Mides: «Jhoannes notvjt» (LP 157, de 1032.04.24); «Johannes Midiz quos vidi et scripsi manu mea conf.» (LP 189, de 1032.07.31); «Johannes notuit» (LP 190, de 1034.07.21); «Johannes presbiter denuntiavit, $\mathrm{A}[n]$ semundus presbiter quos exaravit» (LP 185, de 1038.04.01); Sandino: «Sandinus notuit» (LP 192, de 1045.06.06); Randulfo: «Justo presbitero quo $<\mathrm{s}>$ adsignavit. Randulfus notuit» (LP 520, 
de 1046.01.29); clérigos-notários não-referidos: LP 129 e 161 (1018.01.30), LP 120 (1018), LP 121 (1019.02.28), LP 134 (1019.12.02), LP 144 (1023.09.21), LP 353 (1032.03.13), LP 145 (1034.02.10), LP 115 e 140 (1040.08.13), LP 363 (1041.04.19), LP 521 (1043.05.20), LP 152 (1044.07.22), LP 137 e 148 (1045.09.21), LP 153 (1045.09.21) e LP 369 (1046.08.14).

g) Referências documentais, por ordem cronológica: LP 129 e 161 (1018.01.30), LP 120 (1018), LP 121 (1019.02.28), LP 134 (1019.12.02), LP 142 (1021.11.20), LP 144 (1023.09.21), LP 143 ([1023.09.21]), LP 359 (1025.09.21), LP 109 ([1026-1045]), LP 353 (1032.03.13), LP 157 (1032.04.24), LP 189 (1032.07.31), LP 145 (1034.02.10), LP 190 (1034.07.21), LP 184 (1035.02.23), LP 516 (1035.03.28), LP 147 ([1035].12.04), LP 362 e 511 (1037.03.14), LP 367 (1037.08.04), LP 185 (1038.04.01), LP 186 (1038.04.01), LP 198 (1039.04.22), LP 115 e 140 (1040.08.13), LP 512 (1041.02.28), LP 363 (1041.04.19), LP 521 (1043.05.20), LP152(1044.07.22), LP192(1045.06.06), LP137e 148(1045.09.21), LP 138 e 150 (1045.09.21), LP 153 (1045.09.21), LP 520 (1046.01.29) e LP 369 (1046.08.14).

\section{FlóRIDO}

a) Referências documentais, por título: abbas: LP 93 e 146 (1036.02.22), LP 127 (1041.10.01), LP 84 (1043.09.04), LP 130 e 132 (1047.10.12 ou 13) e LP 110 ([1047].11.19); praepositus: LP 138 e 150 (1045.09.21); título não-referido: LP 53 (1082.01.06).

Obs.: Durante o abaciado de Tudeíldo, Flórido desempenhou funções como prioste do mosteiro de Vacariça (LP 138 e 150, de 1045.09.21). Durante o abaciado de Flórido, Alvito desempenhou funções como prioste no mosteiro de Vacariça (LP 130 e 132, de 1047.10.12 ou 13).

b) Geografia do património adquirido durante o seu abaciado: por doação: Penacova (conc. de Penacova, dist. de Coimbra) (LP 93 
e 146, de 1036.02.22); Agoncide (conc. de St. ${ }^{a}$ Maria da Feira, dist. de Aveiro) (LP 127, de 1041.10.01); Custóias (conc. de Matosinhos, dist. do Porto) e foz do Leça (conc. de Matosinhos, dist. do Porto) (LP 138 e 150, de 1045.09.21); Gulpilhares (conc. de Vila Nova de Gaia, dist. do Porto), Vilar (conc. de Gondomar, dist. do Porto), Negrelos (conc. de Vila Nova de Gaia, dist. do Porto), Arrabalde de Marnel (conc. de Anadia, dist. de Aveiro), Ílhavo (conc. de Ílhavo, dist. de Aveiro), Loure (conc. de Albergaria-a-Velha, dist. de Aveiro), Ossela (conc. de Oliveira de Azeméis, dist. de Aveiro), Aveiro (conc. de Aveiro, dist. de Aveiro), Tarouquela (conc. de Vila Nova de Gaia, dist. do Porto), Recardães (conc. de Águeda, dist. de Aveiro), Antolini (loc. incerta), Nespereira (conc. de Sever do Vouga, dist. de Aveiro), Ferreirós (conc. de Oliveira de Azeméis, dist. de Aveiro), Castro (conc. de St. ${ }^{a}$ Maria da Feira, dist. de Aveiro) e Seixozelo (conc. de Vila Nova de Gaia, dist. do Porto) (LP 130 e 132, de 1047.10.12 ou 13; as referências a Loure, Ossela e Aveiro constam apenas de LP 132) ${ }^{25}$; Esgueira (conc. de Aveiro, dist. de Aveiro) (LP 110, de [1047].11.19); Monsarros (conc. de Anadia,

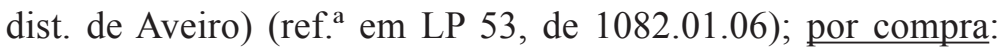
doc. inexistente; por permuta: doc. inexistente.

c) Expressões relativas a actos de produção documental per manum propriam: scripsit: doc. inexistente.

d) Expressões relativas a actos de subscrição documental per manum propriam: adfuit: doc. inexistente; conf.: doc. inexistente; test.: doc. inexistente.

e) Direitos de padroado adquiridos durante o seu abaciado: igreja de S. Pedro de Penacova (conc. de Penacova, dist. de Coimbra) (LP 93 e 146, de 1036.02.22); mosteiro de Soure (conc. de Soure, dist. de Coimbra) (LP 84, de 1043.09.04); mosteiro de S. Salvador de Leça (conc. de Matosinhos, dist. do Porto), mosteiro de

\footnotetext{
${ }^{25}$ Doação conjunta ao abade Flórido e seu prioste, Alvito.
} 
S. Martinho de Anta (conc. de Espinho, dist. de Aveiro) e mosteiro de S. Romão de Vermoim (conc. da Maia, dist. do Porto) (LP 138 e 150, de 1045.09.21); igreja de S. Miguel de Recardães (conc. de Águeda, dist. de Aveiro), igreja de St. ${ }^{a}$ Maria de Fermentões (conc. de Águeda, dist. de Aveiro) e igreja de S. Martinho de Carvalhais (conc. de Águeda, dist. de Aveiro) (LP 130 e 132, de 1047.10.12 ou 13).

f) Clérigos-notários documentados durante o seu abaciado: Ansemundo: «Ansemundus Notuit» (LP 127, de 1041.10.01); Sandino: «Sandinus quasi presbiter Notuit» (LP 130, de 1047.10.12), com a variante «Onorcus Notuit» (LP 132, de 1047.10.13); clérigos-notários não-referidos: LP 93 e 146 (1036.02.22), LP 84 (1043.09.04) e LP 110 ([1047].11.19).

g) Referências documentais, por ordem cronológica: LP 93 e 146 (1036.02.22), LP 127 (1041.10.01), LP 84 (1043.09.04), LP 138 e 150 (1045.09.21), LP 130 e 132 (1047.10.12 ou 13), LP 110 ([1047].11.19) e LP 53 (1082.01.06).

\section{Alvito}

a) Referências documentais, por título: abbas: LP 141 ([1047].12.20), LP 136 ([1053].01.20), LP 114 e 154 (1055.07.09), LP 88 (1057.01.21), LP 210 (1075.03.18), DC 542 (1077.02.26), LP 53 (1082.01.06), LP 75 ([1082.01.06]), LP 104 (1084.08.15), LP 101 (1086.03.25) e LP 334 (s.d.); praepositus e presbyter: LP 130 e 132 (1047.10.12 ou 13).

Obs.: Alvito foi o primeiro abade a exprimir a sua condição de clérigo, utilizando o título de presbyter, no mosteiro de Vacariça (LP 130 e 132, de 1047.10.12 ou 13). Durante o abaciado de Flórido, Alvito desempenhou funções como prioste no mosteiro de Vacariça (LP 130 e 132, de 1047.10.12 ou 13). Durante o abaciado de Alvito, Ildras desempenhou funções como prioste no mosteiro 
de Leça (LP 114 e 154, de 1055.07.09). Ildras deve provavelmente identificar-se com o presbítero homónimo, residente no mosteiro de Lorvão, que doou a esta comunidade monástica os casais e as vinhas que herdara da mãe em Vila Cova, em 1051 (DC 379, de 1051.08.07). É possível que Ildras tenha feito a profissão monástica em Lorvão, antes de seguir para Leça para aí desempenhar funções como prioste.

Como abade do mosteiro de Vacariça: LP 130 e 132 (1047.10.12 ou 13), LP 141 ([1047].12.20), LP 136 ([1053].01.20), LP 114 e 154 (1055.07.09), LP 88 (1057.01.21), LP 53 (1082.01.06), LP 75 ([1082.01.06]), LP 104 (1084.08.15), LP 101 (1086.03.25) e LP 334 (s.d.).

Como abade do mosteiro de Leça: LP 210 (1075.03.18) e DC 542 (1077.02.26).

b) Geografia do património adquirido durante o seu abaciado: por doação: Gulpilhares (conc. de Vila Nova de Gaia, dist. do Porto), Vilar (conc. de Gondomar, dist. do Porto), Negrelos (conc. de Vila Nova de Gaia, dist. do Porto), Arrabalde de Marnel (conc. de Anadia, dist. de Aveiro), Ílhavo (conc. de Ílhavo, dist. de Aveiro), Loure (conc. de Albergaria-a-Velha, dist. de Aveiro), Ossela (conc. de Oliveira de Azeméis, dist. de Aveiro), Aveiro (conc. de Aveiro, dist. de Aveiro), Tarouquela (conc. de Vila Nova de Gaia, dist. do Porto), Recardães (conc. de Águeda, dist. de Aveiro), Antolini (loc. incerta), Nespereira (conc. de Sever do Vouga, dist. de Aveiro), Ferreirós (conc. de Oliveira de Azeméis, dist. de Aveiro), Castro (conc. de St. ${ }^{a}$ Maria da Feira, dist. de Aveiro) e Seixozelo (conc. de Vila Nova de Gaia, dist. do Porto) (LP 130 e 132, de 1047.10.12 ou 13; as referências a Loure, Ossela e Aveiro constam apenas de LP 132) 26; Paredes (conc. de Sever do Vouga, dist. de Aveiro) e Paradela (conc. de Sever do Vouga, dist. de Aveiro) (LP

${ }^{26}$ Doação conjunta ao abade Flórido e seu prioste, Alvito. 
141, de [1047].12.20); Real (conc. de Matosinhos, dist. do Porto) e Gondivai (conc. de Matosinhos, dist. do Porto) (LP 114 e 154, de 1055.07.09) 27; Paçô (conc. de Sever do Vouga, dist. de Aveiro), St. ${ }^{a}$ Cruz (conc. de Vale de Cambra, dist. de Aveiro) e Barreiros (conc. de Sever do Vouga, dist. de Aveiro) (LP 88, de 1057.01.21); Horta (conc. de Anadia, dist. de Aveiro) (LP 101, de 1086.03.25); por compra: doc. inexistente; por permuta: doc. inexistente.

Obs.: É possível que date do abaciado de Alvito o procedimento de aquisição de bens em Recarei que motivou um litígio entre o abade e Paio Guterres, em 1075 (LP 210, de 1075.03.18). É ainda possível que os bens de Monsarros, reivindicados simultaneamente pelo abade e João Justes, em 1082, tenham sido doados ao mosteiro durante o abaciado de Flórido (LP 53, de 1082.01.06; cf. LP 75, de [1082.01.06]). Desconhecemos a data de integração no património monástico do casal emprazado pelo mosteiro a Alvito e sua mulher, Composta, em 1084 (LP 104, de 1084.08.15). Apesar de não termos qualquer informação adicional sobre a data de aquisição destas propriedades, os documentos que as mencionam pela primeira vez podem atribuir-se com segurança ao abaciado de Alvito.

c) Direitos de padroado adquiridos durante o seu abaciado: igreja de S. Miguel de Recardães (conc. de Águeda, dist. de Aveiro), igreja de St. ${ }^{a}$ Maria de Fermentões (conc. de Águeda, dist. de Aveiro) e igreja de S. Martinho de Carvalhais (conc. de Águeda, dist. de Aveiro) (LP 130 e 132, de 1047.10.12 ou 13); igreja de Pedroso (conc. de St. ${ }^{a}$ Maria da Feira, dist. de Aveiro), igreja de Manhouce (conc. de St. ${ }^{a}$ Maria da Feira, dist. de Aveiro), igreja de Escapães (conc. de St. ${ }^{a}$ Maria da Feira, dist. de Aveiro) e igreja de Agoncide (conc. de St. ${ }^{a}$ Maria da Feira, dist. de Aveiro) (LP 136, de [1053].01.20).

${ }^{27}$ Doação conjunta ao abade Alvito e seu prioste, Ildras, dirigida simultaneamente aos mosteiros de S. Vicente de Vacariça e S. Salvador de Leça. 
Obs.: É possível que date do abaciado de Alvito o procedimento de incorporação de direitos de padroado sobre a igreja de St. ${ }^{a}$ Eufémia, referida pela primeira vez como propriedade do mosteiro durante o seu governo (LP 334, s.d.).

d) Expressões relativas a actos de produção documental per manum propriam: scripsit: doc. inexistente.

e) Expressões relativas a actos de subscrição documental per manum propriam: adfuit: doc. inexistente; conf: DC 542 (1088.02.26); test.: doc. inexistente.

f) Clérigos-notários documentados durante o seu abaciado: Sandino: «Sandinus quasi presbiter Notuit» (LP 130, de 1047.10.12), com a variante «Onorcus Notuit» (LP 132, de 1047.10.13); Guterre: «Guterre quasi presbiter Notuit» (LP 141, de [1047].12.20); David: «Dauid Notuit» (LP 136, de [1053].01.20); Randulfo: «Randulfus presbiter Notuit» (LP 114 e 154, de 1055.07.09); Arigo Todereis: «Frater Arigus Toderidiz notuit» (LP 210, de 1075.03.18); Gondesendo: "Gundesindus presbiter Notuit» (LP 53, de 1082.01.16); Froia: «Froia presbiter Notuit» (LP 104, de 1084.08.15); Ero: «Ervs presbiter qui hanc Notuit» (LP 101, de 1086.03.25); Gonçalo: «Gundisalvus notuit» (LP 334, s.d.); clérigos-notários não-referidos: LP 88 (1057.01.21) e LP 75 ([1082.01.06]).

g) Referências documentais, por ordem cronológica: LP 130 e 132 (1047.10.12 ou 13), LP 141 ([1047].12.20), LP 136 ([1053].01.20), LP 114 e 154 (1055.07.09), LP 88 (1057.01.21), LP 210 (1075.03.18), DC 542 (1077.02.26), LP 53 (1082.01.06), LP 75 ([1082.01.06]), LP 104 (1084.08.15), LP 101 (1086.03.25) e LP 334 (s.d.).

\section{Jỗo [MIDES]?}

a) Referências documentais, por título: título não-referido: LP 86 (1047.12.20). 
Obs.: Não é certo que João tenha desempenhado funções como abade no mosteiro de Vacariça. Embora não seja nunca mencionado nessa condição, parece ter exercido uma certa preeminência sobre a comunidade monástica. É possível que tenha desempenhado funções como abade interino durante um breve período de tempo logo após a morte de Tudeíldo, ocorrida em 1046, visto ser citado nessa condição apenas em 1047. É ainda possível que se identifique com João a figura homónima que, sob o patronímico Mides, desempenhou funções como clérigo-notário durante o abaciado de Tudeíldo (LP 157, de 1032.04.24; LP 189, de 1032.07.31; LP 190, de 1034.07.21).

b) Geografia do património adquirido durante o seu [abaciado]: doc. inexistente.

c) Direitos de padroado adquiridos durante o seu [abaciado]: doc. inexistente.

d) Expressões relativas a actos de produção documental per manum propriam: scripsit: doc. inexistente.

e) Expressões relativas a actos de subscrição documental per manum propriam: adfuit: doc. inexistente; conf: doc. inexistente; test.: doc. inexistente.

f) Clérigos-notários documentados durante o seu [abaciado]: doc. inexistente.

g) Referências documentais, por ordem cronológica: LP 86 (1047.12.20).

7. RAMIRo

a) Referências documentais, por título: abbas: LP 372 (1086.08.11); prior: LP 33 (1087.03.14), LP 119 ([1087-1091]) e LP 398 (1088.01.03); abbas, prior e ducator: LP 131 (1093.07.09). 
Obs.: Ramiro foi o primeiro abade a utilizar os títulos de prior e ducator no mosteiro de Vacariça (LP 33, de 1087.03.14, para prior; LP 131, de 1093.07.09, para ducator). Durante o abaciado de Ramiro, Cid Davides desempenhou funções como prioste no mosteiro de Leça (LP 131, de 1093.07.09). Cid Davides chegou a subscrever diplomas de doação ao mosteiro de Vacariça (LP 114 e 154, de 1055.07.09).

b) Geografia do património adquirido durante o seu abaciado: por doação: Marmeleira (conc. de Coimbra, dist. de Coimbra) (LP 372, de 1086.08.11; o documento contempla ainda uma quintã, doada à igreja de S. Salvador de Coimbra); por compra: doc. inexistente; por permuta: doc. inexistente.

Obs.: Foi durante o abaciado de Ramiro que se procedeu ao reconhecimento das propriedades que o mosteiro de Vacariça detinha, por doação, em Recardães, por ordem do cônsul Sesnando Davides, com o objectivo de se esclarecer a contestação de Eusébio, abade do mosteiro de Lorvão, à posse desses bens pela comunidade monástica (LP 119, de [1087-1091]). Este documento não menciona a aquisição de novos bens por Ramiro, referindo apenas os que haviam sido previamente incorporados no património monástico, designadamente a doação do presbítero Zalama ao abade Emilião (LP 123, de 1018.01.12). Foi ainda durante o abaciado de Ramiro que se produziu um documento fazendo prova da aceitação, por quatro leigos, das condições de emprazamento de umas vinhas em terrenos do mosteiro de Leça, mas pertencentes ao mosteiro de Vacariça, que este pretendia recuperar, ficando a terça parte dos bens auferidos a pertencer à comunidade monástica (LP 131, de 1093.07.09).

c) Direitos de padroado adquiridos durante o seu abaciado: doc. inexistente.

d) Expressões relativas a actos de produção documental per manum propriam: scripsit: LP 33 (1087.03.14). 
e) Expressões relativas a actos de subscrição documental per manum propriam: adfuit: LP 398 (1088.01.03); conf: LP 372 (1086.08.11), LP 398 (1088.01.03) e LP 131 (1093.07.09); test.: doc. inexistente.

f) Clérigos-notários documentados durante o seu abaciado: $\underline{\text { Pedro: }}$ «Petrus notuit» (LP 372, de 1086.08.11); Diogo: «Didacus presbiter Notuit» (LP 131, de 1093.07.09); clérigo-notário não-referido: LP 119 ([1087-1091]).

g) Referências documentais, por ordem cronológica: LP 372 (1086.08.11), LP 33 (1087.03.14), LP 119 ([1087-1091]), LP 398 (1088.01.03) e LP 131 (1093.07.09).

8. Salomão

a) Referências documentais, por título: abbas e praepositus: LP 255 (1090.08.16); prior e presbyter: LP 160 (1091.08.02); prior: LP 550 (1092.05), LP 41 (1093.02.27) e LP 77 (1098.12.17); rector e presbyter: LP 173 (1094.02.24); abbas: LP 174 (1095.03.03), LP 125 (1099.03.14), LP 108 (1099.09.15), LS 166 (1137.12) (?) e LS 167 (1137.12) (?).

Obs.: Salomão foi o primeiro abade a utilizar o título de rector no mosteiro de Vacariça (LP 173, de 1094.02.24). Durante o abaciado de Salomão, Godinho desempenhou funções como prioste no mosteiro de Leça.

Como abade do mosteiro de Vacariça: LP 255 (1090.08.16), LP 160 (1091.08.02), LP 550 (1092.05), LP 41 (1093.02.27), LP 77 (1098.12.17), LP 173 (1094.02.24), LP 174 (1095.03.03) e LP 108 (1099.09.15).

Como abade do mosteiro de Trezói: LP 77 (1098.12.17). 
b) Geografia do património adquirido durante o seu abaciado: por doação: S. Martinho de Pinheiro (conc. de Coimbra, dist. de Coimbra) (LP 255, de 1090.08.16); por compra: Aldoar (conc. do Porto, dist. do Porto) (LP 125, de 1099.03.14); por permuta: doc. inexistente.

c) Direitos de padroado adquiridos durante o seu abaciado: igreja de S. Martinho de Pinheiro (conc. de Coimbra, dist. de Coimbra) (LP 174, de 1095.03.03) $)^{28}$.

d) Expressões relativas a actos de produção documental per manum propriam: scripsit: LP 41 (1093.02.27).

e) Expressões relativas a actos de subscrição documental per manum propriam: adfuit: LP 173 (1094.02.24); conf: doc. inexistente; test.: LP 255 (1090.08.16), LS 166 (1137.12) (?) e LS 167 (1137.12) (?).

f) Clérigos-notários documentados durante o seu abaciado: Daniel: «Daniel presbiter notuit» (LP 255, de 1090.08.16); Fromarigo: «Fromarigus Notuit» (LP 160, de 1091.08.02); «Fromarigus scripsit» (LP 174, de 1095.03.03); Soeiro: «Suarius presbiter Notuit» (LP 41, de 1093.02.27); Gonçalo: «Gvndisaluus Notuit» (LP 125, de 1099.03.14); Gondesendo: "Gondesindus presbiter Notuit» (LP 108, de 1099.09.15); clérigo-notário não-referido: LP 77 (1098.12.17).

g) Referências documentais, por ordem cronológica: LP 255 (1090.08.16), LP 160 (1091.08.02), LP 550 (1092.05), LP 41 (1093.02.27), LP 173 (1094.02.24), LP 174 (1095.03.03), DC 825 (1096.02.14), LP 77 (1098.12.17), LP 125 (1099.03.14), LP 108 (1099.09.15), LS 166 (1137.12) (?) e LS 167 (1137.12) (?).

${ }^{28}$ Doação dirigida simultaneamente à igreja de S. Salvador de Coimbra e ao mosteiro de S. Vicente de Vacariça. 